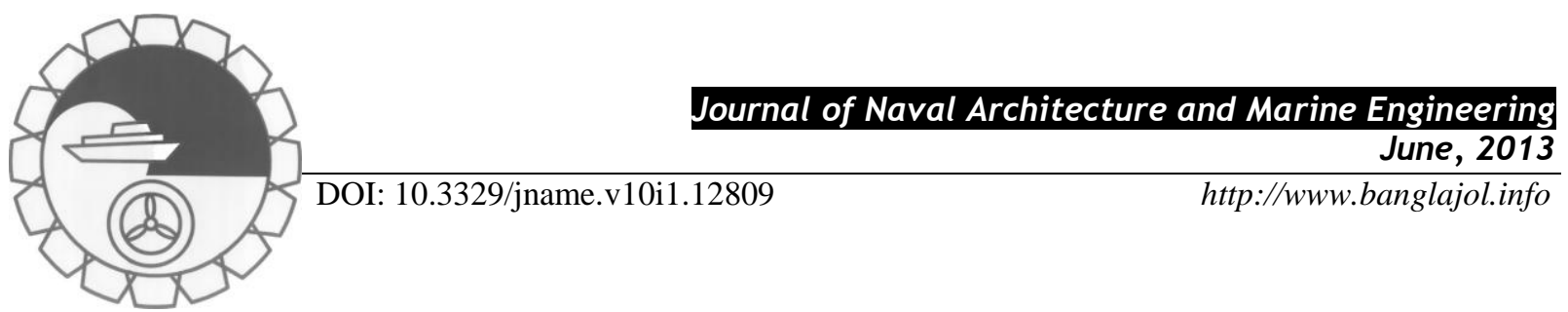

\title{
EFFECTS OF VISCOUS DISSIPATION ON NATURAL CONVECTION FLOW ALONG A SPHERE WITH RADIATION AND HEAT GENERATION Salina Aktar ${ }^{1}$, Mahmuda Binte Mostafa Ruma ${ }^{2}$ and M. A. Alim ${ }^{3}$
}

${ }^{1}$ Department of Natural Science, Stamford University Bangladesh, Siddeswari, Dhaka -1216, Bangladesh, Email: salina854@gmail.com

${ }^{2}$ Department of Engineering \& Technology, Eastern University. Dhanmondi,Dhaka-1208, Bangladesh, Email: ruma_11@live.com

${ }^{3}$ Department of Mathematics, Bangladesh University of Engineering and Technology, Dhaka-1000, Bangladesh, Email: maalim@math.buet.ac.bd

\begin{abstract}
:
The effects of viscous dissipation on free convection flow along a sphere with radiation and heat generation have been investigated in this paper. The governing equations with associated boundary conditions for this phenomenon are converted to dimensionless forms using a suitable transformation. The transformed non-linear equations are then solved using the implicit finite difference method. Numerical results of the velocity and temperature profiles, skin friction coefficient and surface temperature profiles for different values of the of viscous dissipation parameter Vd ( 0.0 to 4.0), radiation parameter $R d$ (0.0 to 1.0), the Prandlt number $\operatorname{Pr}(0.72$ to 7.0$)$ and the heat generation parameter $Q$ (0.2 to 0.6) are presented graphically. Detailed discussion is given for the effects of the aforementioned parameters. Significant effect is found in the velocity and temperature profiles, skin friction coefficient and surface temperature distribution for the Prandtl number, heat generation and radiation parameters.
\end{abstract}

Keywords: Natural convection, viscous dissipation, radiation, heat generation, skin-friction, Nusselt number.

\begin{tabular}{|c|c|c|c|}
\hline \multicolumn{2}{|c|}{ NOMENCLATURE } & $V d$ & viscous dissipation parameter \\
\hline$a$ & radius of sphere & \multicolumn{2}{|c|}{ Greek symbols } \\
\hline$a_{r}$ & Rosseland mean absorption co-efficient & $u, v$ & velocity components \\
\hline$C_{f x}$ & local skin-friction coefficient & $\hat{u}, \hat{v}$ & dimensionless velocity components \\
\hline$C p$ & specific heat at constant pressure & $x, y$ & cartesian co-ordinates \\
\hline$f$ & dimensionless stream function & $\hat{x}, \hat{y}$ & dimensionless cartesian co-ordinates \\
\hline$G r$ & Grashof number & $\beta$ & volumetric coefficient \\
\hline$g$ & acceleration due to gravity & $\xi$ & dimensionless coordinates along the surface \\
\hline$k$ & thermal conductivity & $\eta$ & dimensionless coordinates normal to the surface \\
\hline$N u_{x}$ & local Nusselt number & $\theta$ & dimensionless temperature \\
\hline $\operatorname{Pr}$ & Prandtl number & $\theta_{w}$ & surface temperature parameter \\
\hline$Q$ & heat generation parameter & $\mu$ & viscosity of the fluid \\
\hline$q_{c}$ & conduction heat flux & $v$ & kinematic viscosity \\
\hline$q_{r}$ & radiation heat flux & $\rho$ & density of the fluid \\
\hline$R d$ & radiation parameter & $\sigma$ & Stephan-Boltzman constant \\
\hline$r$ & radial distance & $\sigma_{s}$ & scattering coefficient \\
\hline$T$ & temperature of the fluid & $\tau_{w}$ & shearing stress \\
\hline$T_{\infty}$ & temperature of the ambient fluid & $\psi$ & stream function \\
\hline
\end{tabular}




\section{Introduction}

Convection is the mode of energy transfer between a solid surface and the adjacent liquid or gas that is in motion and it involves the combined effects of conduction and fluid motion. The faster the fluid motion, the greater the heat transfer due to convection. Free convection flow is often encountered in cooling of nuclear reactors or in the study of the structure of stars and planets. The study of temperature and heat transfer is of great importance to the engineers because of its almost universal occurrence in many branches of science and engineering. Although heat transfer is most important for the proper sizing of fuel elements in the nuclear reactors cores to prevent burnout by Sutton and Biblarz (2001). The viscous dissipation effect plays an important role in natural convection in various devices which are subjected to large decleration or which operate at high rotational speeds and also in strong gravitational field processes on large scales(on large planets), in geological process and in nuclear engineering in connection with the cooling of reactors. Possible heat generation effects may alter the temperature distribution and therefore the particle deposition rate. This may occur in such applications related to nuclear reactor cores, fire and combustion modeling, electronic chips and semiconductor wafers. Along with the free convection flow, the phenomenon of the boundary layer flow of an incompressible fluid along a sphere in presence of viscous dissipation, radiation and heat generation is important as well as exceptional because of their applications in nuclear engineering in connection with the cooling of reactors. The discussion and analysis of natural convection flows, viscous dissipation effect in presence of radiation and heat generation are generally ignored but here we have considered the effect of viscous dissipation on natural convection flow along a sphere with radiation and heat generation.

The influence and importance of viscous stress work effects in laminar flows have been examined by Gebhart (1962), Gebhart and Mollendorf (1969). In both of the investigations special flows over semi-infinite flat surface parallel to the direction of body force were considered. Gebhart (1962) considered flows generated by the plate surface temperature, which vary as power of $\xi$ (the distance along the plate surface from the leading edge),and Mollendrof (1969) considered flows generated by plate surface temperature,which vary exponentially in $\xi$. Zakerullah (1972) has been investigated the viscous dissipation and pressure work effects in axisymmetric natural convection flows. Huang and Chen (1987) and Nazar et al. (2002) considered the free convection boundary layer on an isothermal sphere in a micro polar fluid. Molla et al. (2004), (2005) have studied the problem of natural convection flow along a vertical wavy surface and Magneto hydrodynamics natural convection flow on a sphere with uniform surface temperature in presence of heat generation or absorption. Alam et al. (2006) have analyzed pressure stress work and viscous dissipation effects in natural convection flow along a vertical flat plate with heat conduction. Molla et al. (2006) have studied natural convection flow from an isothermal horizontal cylinder in presence of heat generation. There are also some works of viscous dissipation effects on natural boundary layer flow from a sphere either heat generation or radiation. Alam et al. (2006), (2007) has described viscous dissipation effects on MHD natural convection flow along a sphere in presence of heat generation. Effects of radiation on natural convection flow along a sphere with uniform heat flux have been analyzed by Akhter (2008). Viscous dissipation effects on natural convection flow along a sphere with radiation heat loss has been studied by Alim(2008). Miraj et al. (2010) studied effect of radiation on natural convection flow on a sphere in presence of heat generation. Tania et al. (2010) has investigated the effects of radiation, heat generation and viscous dissipation on MHD free convection flow along a stretching sheet. Ali et al. (2011) investigated conjugate effects of Radiation and Joule Heating on Magnetohydrodynamic free convection flow along a sphere with heat generation. Miraj et al. (2011) analyzed effects of pressure work and on natural convection flow around a sphere with heat generation. At last Ibrahim (2013) studied the effect of chemical reaction and radiation on MHD free convection flow along a stretching surface in presence of viscous dissipation and heat generation. From all the analysis, the effects of radiation, heat generation and viscous dissipation has been discussed separately or simultaneously on MHD free convection flow and in another surface except on the sphere.

To the best of our knowledge, the effect of viscous dissipation on natural convection flow along a sphere with radiation and heat generation has not been studied yet and the present work demonstrates the issue. In the present work, we propose to investigate the viscous dissipation effect on free convection flow along an isothermal sphere in presence of radiation and heat generation. Under the usual Boussinesq approximation, the governing partial differential equations are reduced to local non-similar partial differential forms by adopting appropriate transformations. The transformed boundary layer equations are solved numerically using the Keller box (implicit finite difference) method, Cebeci and Bradshaw (1984) along with Newton's linearization approximation method in the entire region. Numerical results have been obtained in terms of local skin-friction, rate of heat transfer, velocity profiles, temperature profiles for a selection of relevant physical parameters 
consisting of viscous dissipation parameter $V d$, radiation parameter $R d$, the Prandlt number $P r$ and the heat generation parameter $Q$.

\section{Mathematical Formulation}

The natural convection boundary layer flow from an isothermal sphere of radius a, which is immersed in a viscous and incompressible optically dense fluid with radiation and heat generation is considered. It is assumed that the constant temperature at the surface of the sphere is $T_{w}$, where $T_{w}>T_{\infty}$. Here $T_{\infty}$ is the ambient temperature of the fluid, $T$ is the temperature of the fluid in the boundary layer. The coordinates system and the configuration are shown in Fig. 1.

According to the above assumption, the governing for continuity, momentum and energy take the following

$$
\begin{aligned}
& \text { form } \frac{\partial}{\partial \hat{x}}(r \hat{u})+\frac{\partial}{\partial \hat{y}}(r \hat{v})=0 \\
& \hat{u} \frac{\partial \hat{u}}{\partial \hat{x}}+\hat{v} \frac{\partial \hat{u}}{\partial \hat{y}}=v \frac{\partial^{2} \hat{u}}{\partial \hat{y}^{2}}+g \beta\left(T-T_{\infty}\right) \sin \left(\frac{\hat{x}}{a}\right)
\end{aligned}
$$

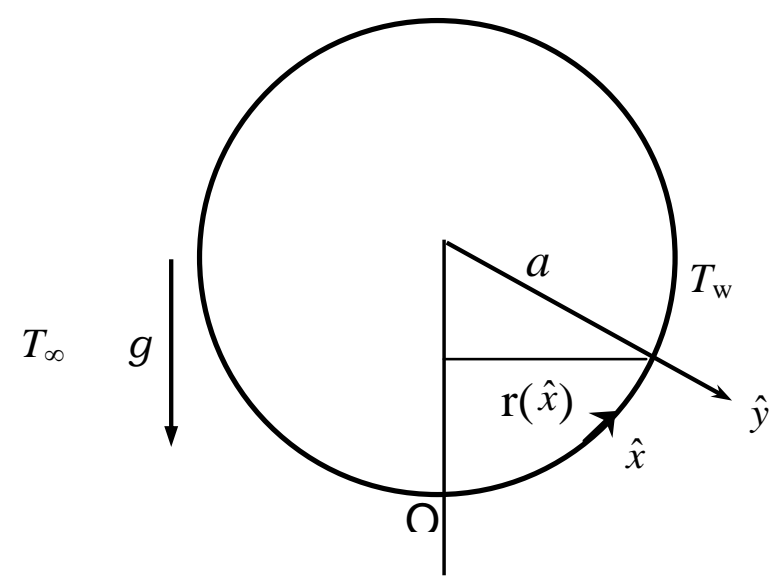

Fig. 1: Physical model and coordinate system

$$
\hat{u} \frac{\partial T}{\partial \hat{x}}+\hat{v} \frac{\partial T}{\partial \hat{y}}=\frac{k}{\rho C_{p}}\left[\frac{\partial^{2} T}{\partial \hat{y}^{2}}-\frac{1}{k} \frac{\partial q_{r}}{\partial \hat{y}}\right]+\frac{v}{\rho C_{P}}\left(\frac{\partial \hat{u}}{\partial \hat{y}}\right)^{2}+\frac{Q_{0}}{\rho C_{p}}\left(T-T_{\infty}\right)
$$

where $r(\hat{x})=a \sin (\hat{x} / a)$ is the radial distance from the centre of the sphere, $g$ is the acceleration due to gravity, $\beta$ is the coefficient of thermal expansion, $v$ is the kinematics viscosity, $T$ is the fluid temperature, $C_{p}$ is the specific heat at constant pressure, $\rho$ is the density and $q_{r}$ is the radiative heat flux. The amount of heat generated or absorbed per unit volume is $Q_{0}\left(\mathrm{~T}-\mathrm{T}_{\infty}\right), Q_{0}$ being a constant, which may take either positive or negative. The source term represents the heat generation when $Q_{0}>0$ and the heat absorption when $Q_{0}<0$.

In order to reduce the complexity of the problem and to provide a means of comparison with future studies that will employ a more detail representation for the radiative heat flux, the optically thick radiation limit is considered in this study. Thus the Rossel and diffusion approximation proposed by Siegen and Howell (1972) is given by simplified radiation heat flux term as:

$q_{r}=-\frac{4 \sigma}{3\left(a_{r}+\sigma_{s}\right)} \frac{\partial T}{\partial \hat{y}^{4}}$ 
Where, $a_{r}$ is the Rossel and mean absorption co-efficient, $\sigma_{s}$ is the scattering co-efficient and $\sigma$ is the StephanBoltzman constant.

The boundary conditions for Equations (1) to (3) are:

$\hat{u}=\hat{v}=0, T=T_{w}$ at $\hat{y}=0$

$\hat{u} \rightarrow 0, T \rightarrow T_{\infty}$ at $\hat{y} \rightarrow \infty$

Where, $T_{w}$ is the surface temperature of the sphere and $T_{\infty}$ is the ambient temperature of the fluid with $T_{w}>T_{\infty}$.

The above equations are non-dimensionalized using the following new variables:

$\xi=\frac{\hat{x}}{a}, \eta=G r^{\frac{1}{4}} \frac{\hat{y}}{a}, \mathrm{u}=\frac{\mathrm{a}}{v} \mathrm{G}_{\mathrm{r}}^{-1 / 2} \hat{\mathrm{u}}, v=\frac{\mathrm{a}}{v} \mathrm{G}_{\mathrm{r}}^{-1 / 4} \hat{v}$,

$\theta=\frac{T-T_{\infty}}{T_{w}-T_{\infty}}, G r=\frac{g \beta\left(T_{w}-T_{\infty}\right) a^{3}}{v^{2}}$,

$\theta_{w}=\frac{T_{w}}{T_{\infty}}, \Delta=\theta_{w}-1=\frac{T_{w}}{T_{\infty}}-1=\frac{T_{w}-T_{\infty}}{T_{\infty}}$

Where $G r=g \beta\left(T_{w}-T_{\infty}\right) a^{3} / v^{2}$ is the Grashof number and $\theta$ is the dimensionless temperature and $\theta_{w}$ is the surface temperature parameter.

Substituting variables (5), (6) and (7) in the continuity equation (1), the momentum equation (2) and the energy equation (3) leads to the following non-dimensional equations:

$\frac{\partial}{\partial \xi}(r u)+\frac{\partial}{\partial \eta}(r v)=0$

$u \frac{\partial u}{\partial \xi}+v \frac{\partial u}{\partial \eta}=\frac{\partial^{2} u}{\partial \eta^{2}}+\theta \sin \xi$

$u \frac{\partial \theta}{\partial \xi}+v \frac{\partial \theta}{\partial \eta}=\frac{1}{\operatorname{Pr}} \frac{\partial}{\partial \eta}\left[\left\{1+\frac{4}{3} R d\left(1+\left(\theta_{w}-1\right) \theta\right)^{3}\right\} \frac{\partial \theta}{\partial \eta}\right]+V d\left(\frac{\partial u}{\partial \eta}\right)^{2}+Q \theta$

Where, $\operatorname{Pr}=\frac{\mu C_{p}}{k}$ is the Prandtl number, $V d=\frac{v^{2} G_{r}}{\rho a^{2} C_{p}\left(T_{w}-T_{\infty}\right)}$ is the viscous dissipation parameter and $R d=\frac{4 \sigma T_{\infty}^{3}}{k\left(a+\sigma_{s}\right)}$ is the radiation parameter.

With the boundary conditions (4) become

$u=v=0, \theta=1$ at $\eta=0$

$u \rightarrow 0, \theta \rightarrow 0$ as $\eta \rightarrow \infty$

To solve Equations (9) and (10) with the help of the following variables

$\psi=\xi r(\xi) f(\xi, \eta), \quad \theta=\theta(\xi, \eta), r(\xi)=\sin \xi$

Where, $\psi(\xi, \eta)$ is the non-dimensional stream function, which is related to the velocity components in the usual way as:

Effects of Viscous Dissipation on Natural Convection Flow along a Sphere with Radiation and Heat Generation 
$u=\frac{1}{r} \frac{\partial \psi}{\partial \eta}$ and $v=-\frac{1}{r} \frac{\partial \psi}{\partial \xi}$

We may proceed to transform the conservation of momentum and energy equations (9) and (10) into the new co-ordinates. To facilitate the transformation, it is useful to have the velocity components explicitly expressed in terms of the new variables. Therefore we obtained

$$
\begin{aligned}
& \frac{\partial^{3} f}{\partial \eta^{3}}+\left(1+\frac{\xi}{\sin \xi} \cos \xi\right) f \frac{\partial^{2} f}{\partial \eta^{2}}-\left(\frac{\partial f}{\partial \eta}\right)^{2}+\theta \frac{\sin \xi}{\xi} \\
&=\xi\left(\frac{\partial f}{\partial \eta} \frac{\partial^{2} f}{\partial \xi \partial \eta}-\frac{\partial f}{\partial \xi} \frac{\partial^{2} f}{\partial \eta^{2}}\right)
\end{aligned}
$$

and

$$
\begin{aligned}
& \frac{1}{\operatorname{Pr}}\left[\frac{\partial}{\partial \eta}\left\{1+\frac{4}{3} R d\left(1+\left(\theta_{w}-1\right) \theta\right)^{3}\right\} \frac{\partial \theta}{\partial \eta}\right]+\left(1+\frac{\xi}{\sin \xi} \cos \xi\right) f \frac{\partial \theta}{\partial \eta} \\
& +V d\left\{\xi \frac{\partial}{\partial \eta}\left(\frac{\partial f}{\partial \eta}\right)\right\}^{2}+Q \theta=\xi\left(\frac{\partial f}{\partial \eta} \frac{\partial \theta}{\partial \xi}-\frac{\partial f}{\partial \xi} \frac{\partial \theta}{\partial \eta}\right)
\end{aligned}
$$

It has been seen that the lower stagnation point of the sphere or $\xi \approx 0$, Equations (12) and (13) reduce to the following ordinary differential equations:

$$
\begin{aligned}
& \frac{\partial^{3} f}{\partial \eta^{3}}+2 f \frac{\partial^{2} f}{\partial \eta^{2}}-\left(\frac{\partial f}{\partial \eta}\right)^{2}+\theta=0 \\
& \frac{1}{\operatorname{Pr}}\left[\frac{\partial}{\partial \eta}\left\{1+\frac{4}{3} \operatorname{Rd}\left(1+\left(\theta_{w}-1\right) \theta\right)^{3}\right\} \frac{\partial \theta}{\partial \eta}\right]+2 f \frac{\partial \theta}{\partial \eta}+Q \theta=0
\end{aligned}
$$

Subject to the boundary conditions

$$
\begin{aligned}
& f=f^{\prime}=0, \theta=1 \text { at } \eta=0 \\
& f^{\prime} \rightarrow 0, \theta \rightarrow 0 \text { as } \eta \rightarrow \infty
\end{aligned}
$$

Where prime denotes the differentiation of the function with respect to $\eta$.

In practical applications, the physical quantities of principle interest are the shearing stress in terms of the local skin-friction coefficients $C_{f x}$ and the rate of heat transfer in terms of local Nusselt number $N u_{x}$, which can be written in non-dimensional form as :

$$
\begin{aligned}
& C_{f x}=\frac{G r^{-3 / 4} a^{2}}{\mu v}\left(\tau_{w}\right) \text { and } N u_{x}=\frac{a G r^{-1 / 4}}{k\left(T_{w}-T_{\infty}\right)}\left(q_{c}+q_{r}\right)_{\hat{y}=0} \\
& \text { where } \tau_{w}=\mu\left(\frac{\partial \hat{u}}{\partial \hat{y}}\right)_{\hat{y}=0}, \quad q_{c}=-k\left(\frac{\partial T}{\partial \hat{y}}\right)_{\hat{y}=0}
\end{aligned}
$$

Where $\tau_{w}$ is the shearing stress and $q_{c}$ is the conduction heat flux, $\mathrm{k}$ being the thermal conductivity and $q_{r}$ is the radiation heat flux. Now 


$$
\begin{aligned}
& \tau_{w}=\mu\left(\frac{\partial \hat{u}}{\partial \hat{y}}\right)_{\hat{y}=0}=\frac{\mu \gamma}{a^{2}} \frac{\xi \partial^{2} f(\xi, 0)}{\partial \eta^{2}} G r^{\frac{3}{4}} \text { and } \\
& q_{c}=-k\left(\frac{\partial T}{\partial \hat{y}}\right)_{\hat{y}=0}=-k \frac{\partial \theta(\xi, 0)}{\partial \eta} \frac{G r^{\frac{1}{4}}}{a}\left(T_{w}-T_{\infty}\right)
\end{aligned}
$$

Using Equations (5), (6) and boundary condition (18) and putting the values of $\tau_{w}$ and $q_{c}$ in (19), we get therefore,

$$
C_{f x}=\xi \frac{\partial^{2} f(\xi, 0)}{\partial \eta^{2}} \quad \text { and } \quad N u_{x}=-\frac{\partial \theta(\xi, 0)}{\partial \eta}
$$

\section{Results and Discussion}

The objective of the present work is to analyze the effect of viscous dissipation on natural convection flow over a sphere in presence of radiation and heat generation. Solutions are obtained for the values of viscous dissipation parameter $V d(0.0,1.0,2.0,3.0,4.0)$, radiation parameter $R d(0.0,0.3,0.5,0.8,1.0)$, the Prandlt number $P r$ $(0.72,1.0,2.0,5.0,7.0)$ and the heat generation parameter $Q(0.2,0.3,0.4,0.5,0.6)$. If we know the values of the functions $f(\eta, \xi), \theta(\eta, \xi)$ and their derivatives for different values of the pertinent parameters then it is possible to calculate the numerical values of the surface temperature $\theta(0, \xi)$ and the velocity gradient(skin frictions) $f^{\prime \prime}(0, \xi)$ at the surface that are important from the physical point of view.

Both the velocity and the temperature profiles accumulate nearly at the some positions of $\eta$, i.e, there is no change of the velocity boundary layer thickness and thermal boundary layer thickness as well. It does not expand for large values of $\eta$.Viscous dissipation parameter $V d$ on the velocity profile and temperature profile increases in Figs. 2(a) and 2 (b). We observe that in Fig. 2(a) the velocity profile increases with the increase of the viscous dissipation parameter $V d$ which indicates that viscous dissipation increases the fluid motion slightly. In Fig. 2 (b) the similar behavior has also been observed for the temperature profile within the similar values of controlling parameters $V d$.

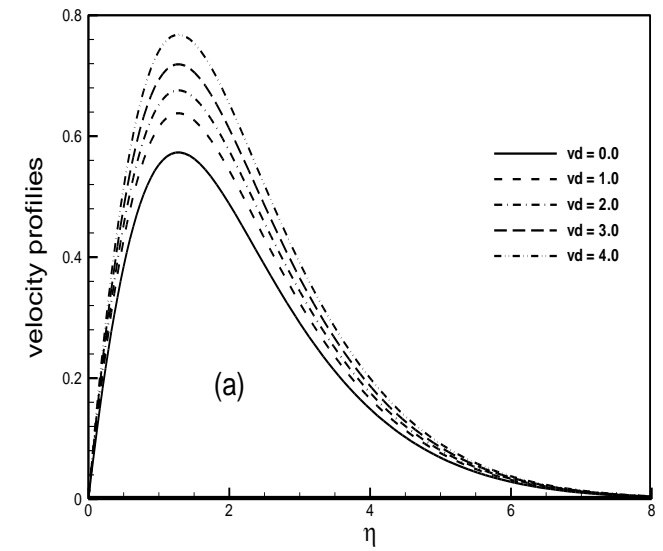

(a)

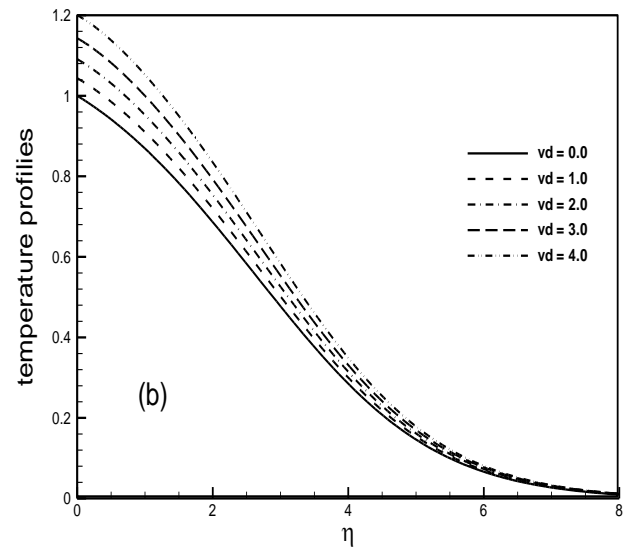

(b)

Fig. 2: (a) Velocity and (b) Temperature profiles against $\eta$ for different values of viscous dissipation parameter $V d$ with $R d=1.0, \operatorname{Pr}=0.72$ and $Q=0.40$.

The effects of varying Prandtl number $\operatorname{Pr}$ on the velocity profile and the dimensionless temperature profile for the viscous dissipation parameter $V d=2.0$, radiation parameter $R d=1.0$ and heat generation parameter $Q=0.40$ 
are shown in Figs. 3(a)-3(b). From Fig. 3(a) we may conclude that the velocity decreases when the value of the Prandtl number Pr increases. But it is seen that near the surface of the sphere the velocity increases considerably and become maximum and then decreases slowly and finally approaches to zero. Whereas in Fig. 3 (b) the temperature profile decreases along with the increase of the Prandtl number $\mathrm{Pr}$. In case of water at $20^{\circ} \mathrm{C}(\mathrm{Pr}$ =7.0), the free laminar boundary shows a sharp decrease compared to the effects in electrolyte solution such as salt water $(\operatorname{Pr}=1.0)$ and air $(\operatorname{Pr}=0.72)$ at $20^{\circ} \mathrm{C}$ and $\operatorname{Pr}=3.0,5.0$ have been used theoretically.

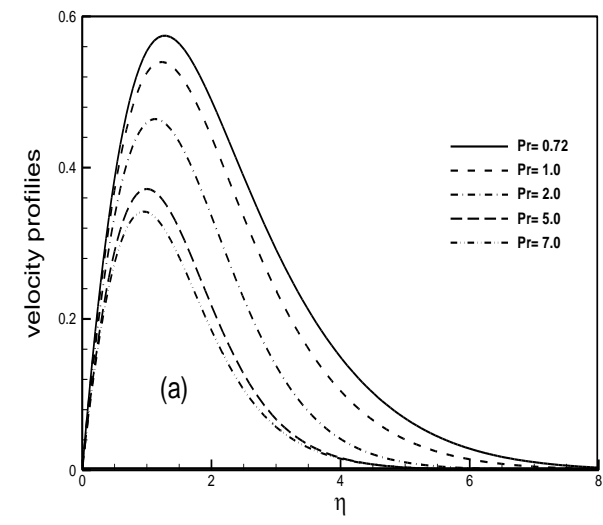

(a)

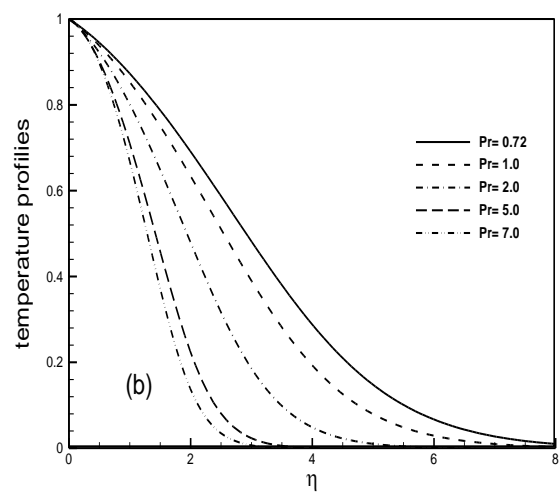

(b)

Fig. 3: (a) Velocity and (b) Temperature profiles against $\eta$ for different values of Prandtl number $\operatorname{Pr}$ with $V d=$ $2.0, R d=1.0$ and $Q=0.40$.

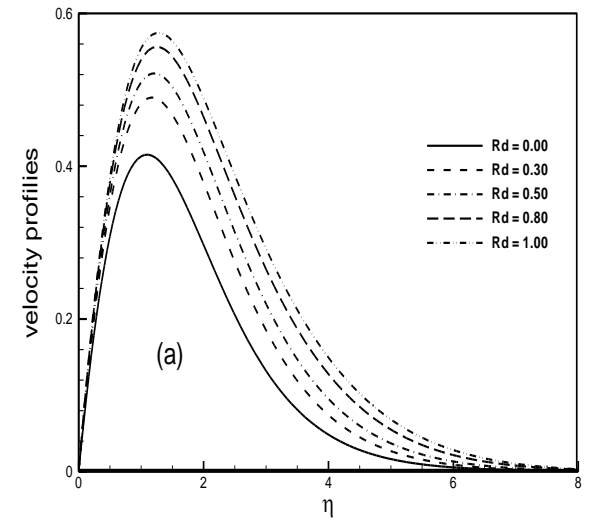

(a)

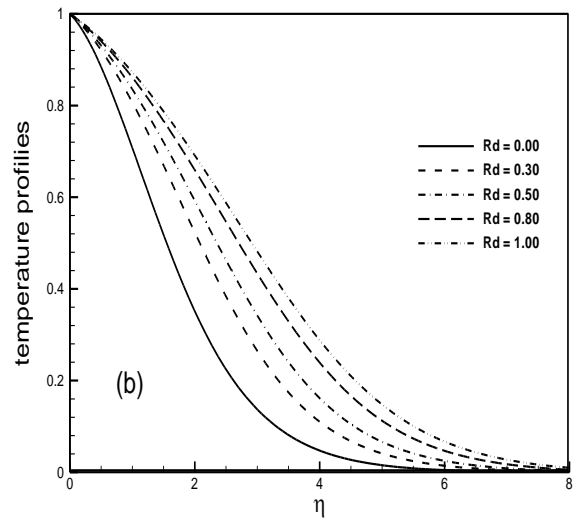

(b)

Fig. 4: (a) Velocity and (b) Temperature profiles against $\eta$ for different values of radiation parameter $R d$ with $V d=2.0, \operatorname{Pr}=0.72$ and $Q=0.40$.

Figs. 4(a) and 4(b) illustrate the velocity and temperature profiles for different values of radiation parameter with $\operatorname{Pr}=0.72, Q=0.4$ and $V d=2.0$. It is observed that velocity profiles increases as the radiation parameter $R d$ increases in Fig. 4(a), which is physically realizable as higher radiation occurs when temperature is higher band eventually velocity rises. From Fig. 4(b), it is seen that the temperature profile also the same as increasing within the boundary layer. Furthermore, the maximum values of the temperature are $0.66363,0.76069,0.78251$, $0.81763,0.82255$ for $R d=0.0,0.3,0.5,0.8,1.0$ respectively and each of which occurs at the surface. It is shown that the temperature increases by $23.94 \%$ when $R d$ increases from 0.0 to 1.0 . This is to be noted that the temperature profiles having the higher values for the increasing value of the radiation parameter and the temperature profiles increases comparatively slower along $\eta$-direction for the lower values of the radiation parameter $R d$ It means that the velocity boundary layer and the thermal boundary layer thickness expand for large values of $R d$. 


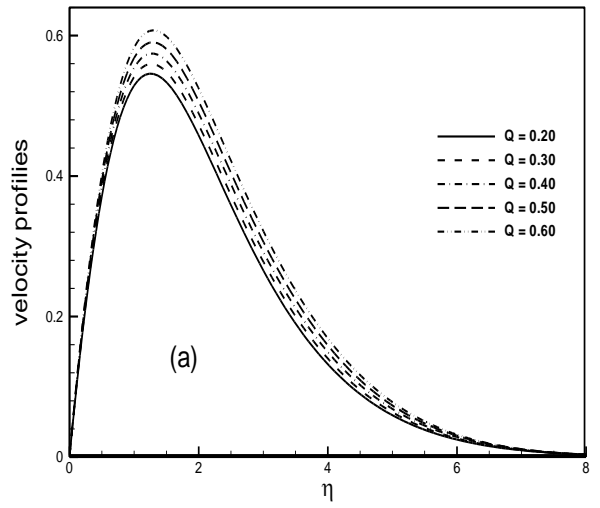

(a)

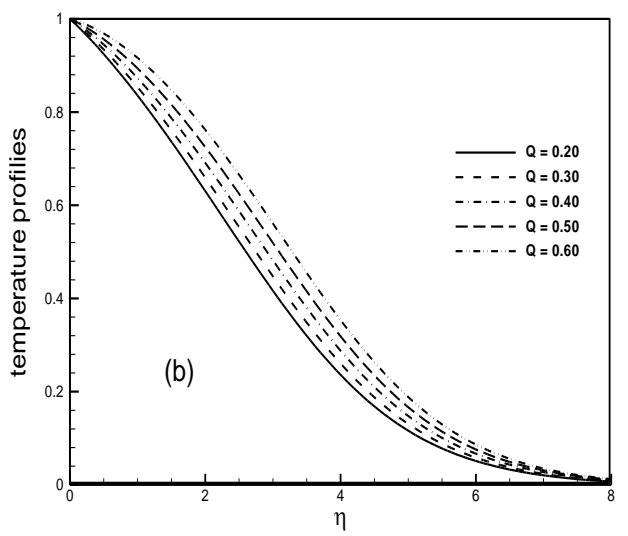

(b)

Fig. 5: (a) Velocity and (b) Temperature profiles against $\eta$ for different values of heat generation parameter $Q$ with $V d=2.0, \operatorname{Pr}=0.72$ and $R d=1.0$.

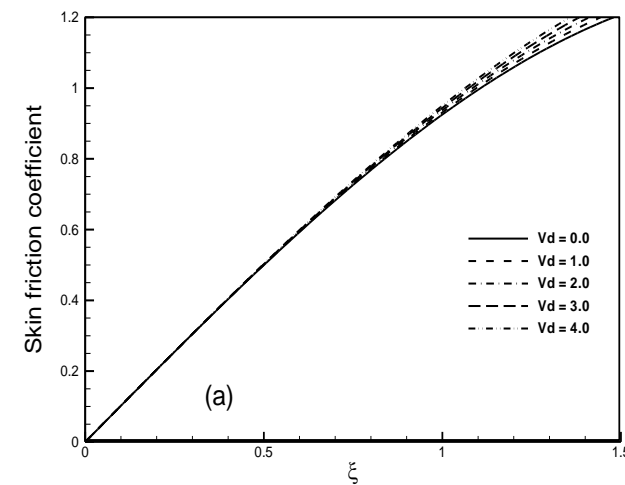

(a)

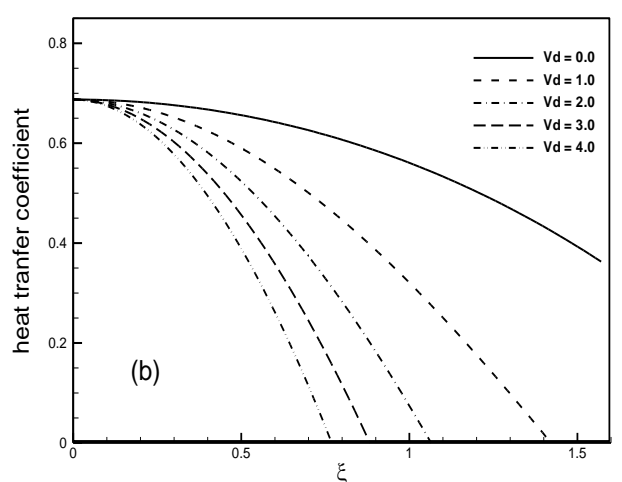

(b)

Fig. 6: (a) Skin friction coefficients and (b) Heat transfer coefficients against $\xi$ for different values of viscous dissipation parameter $V d$ with $R d=1.0, Q=0.40$ and $\operatorname{Pr}=0.72$.

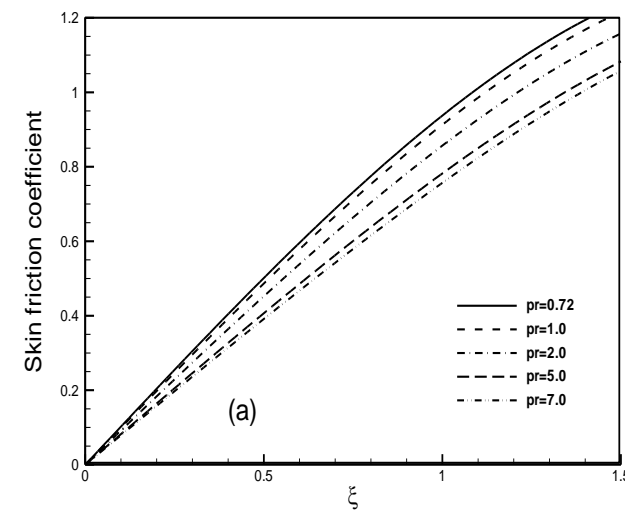

(a)

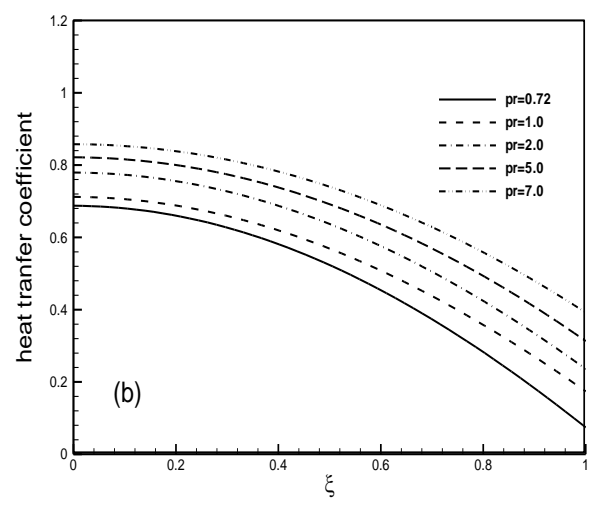

(b)

Fig. 7: (a) Skin friction coefficients and (b) heat transfer coefficients against $\xi$ for different values of Prandtl number $\operatorname{Pr}$ with $V d=2.0, R d=1.0$ and $Q=0.40$. 


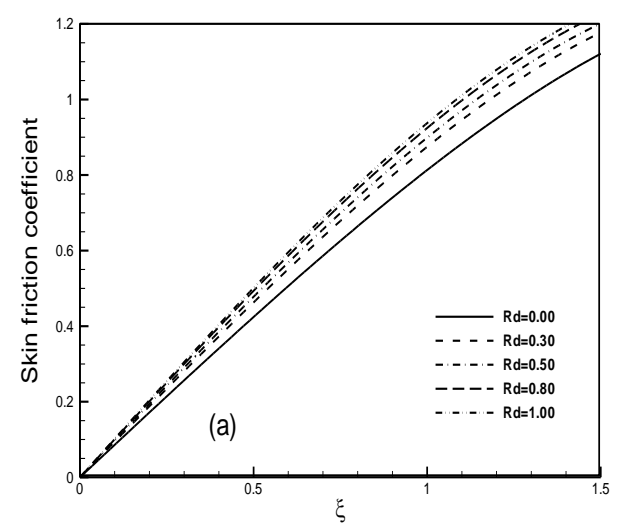

(a)

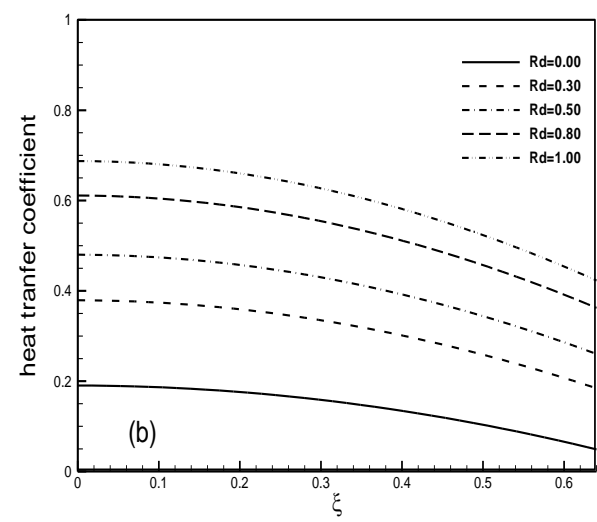

(b)

Fig. 8: (a) Skin friction coefficients and (b) Heat transfer coefficients against $\xi$ for different values of radiation parameter $R d$ with $V d=2.0, \operatorname{Pr}=0.72$ and $Q=0.40$.

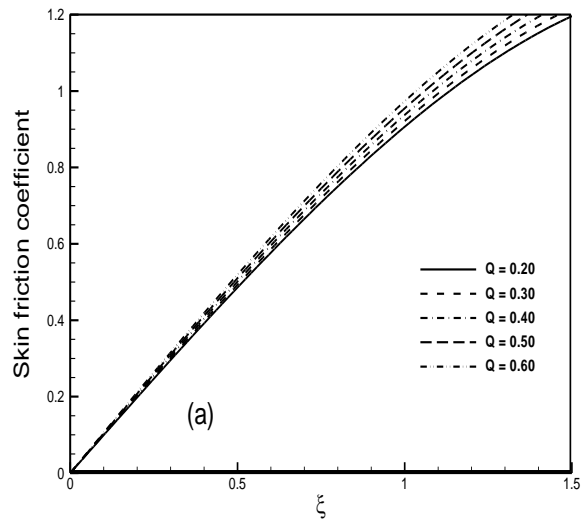

(a)

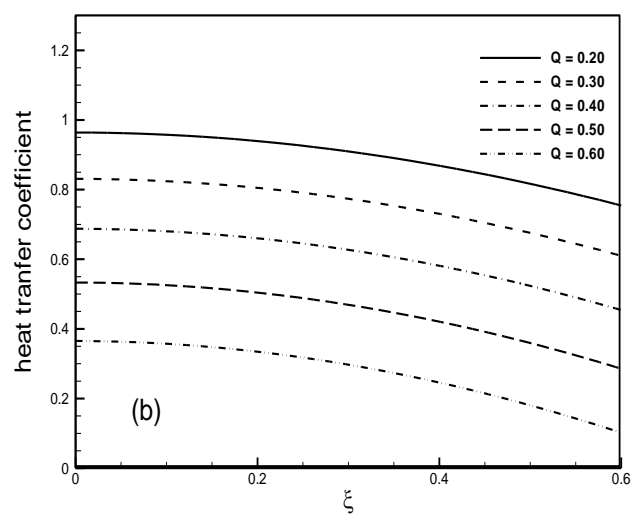

(b)

Fig. 9: (a) Skin friction coefficients and (b) heat transfer coefficients against $\xi$ for different values of heat generation parameter $Q$ with $V d=2.0, \operatorname{Pr}=0.72$ and $R d=1.0$.

Figs. 5 (a) and 5(b) indicate the effects of heat generation parameter $Q$ with $R d=1.0, \operatorname{Pr}=0.72, V d=2.0$ on the velocity profiles and temperature Profiles. Both the velocity and the temperature profiles accumulate nearly at the some positions of $\eta$. i.e. there is no change of the velocity boundary layer thickness and thermal boundary layer thickness as well. Heat generation parameter $Q$ on the velocity profile and temperature profile increases in Figs. 5(a) and 5(b). We observe that in Fig. 5(a) the velocity profile increases with the increase of the heat generation parameter $Q$. The velocity increases as well as its position moves upward the interface with the increasing values of $Q$. While in Fig. 5 (b) the temperature profile increases along with the increase of the heat generation parameter. It means that the velocity boundary layer and the thermal boundary layer thickness expand for large values of $Q$. The maximum values of the velocity are $0.54577,0.55946,0.57448,0.59049$, 0.60760 which occur at $\eta=1.23788$ for 1 st and $2^{\text {nd }}$ maximum values. At $\eta=1.30254$ for $3^{\text {rd }}$, 4th and $5^{\text {th }}$ maximum values. Here it is observed that the velocity increases by $11.32 \%$ as $Q$ increases from 0.20 to 0.60 .

In Figs. 6-9, numerical values of local skin- friction coefficient and the local heat transfer coefficient obtained by finite difference method has been presented for different values of viscous dissipation parameter $V d$, Prandtl number $P r$, radiation parameter $R d$ and heat generation parameter $Q$.

The variation of the local skin friction co-efficient $C_{f x}$ and local rate of heat transfer for different values of $V d$ with $P r=0.72, R d=1.0$ and $Q=0.40$ at different positions of $\xi$ are illustrated in Figs. 6(a) and 6(b) respectively. It is seen in Fig. 6(a), the skin friction coefficient $C_{f x}$ decreases first up to the certain position of $\xi$ and then it increases as the viscous dissipation parameter $V d$ increases. It is seen that an increase in the 
dissipative heat is associated with the enhancing of the skin friction co-efficient. In Fig. 6(b), the rate of heat transfer $N u_{x}$ increases up to the certain position of $\xi$ and also the rate of heat transfer $N u_{x}$ decreases for increasing values of the viscous dissipation parameter $V d$. Frictional force at the surface of the sphere becomes much lower towards the downstream for higher values of $V d$ and the rate of heat transfer as shown in Fig. 6(b) gradually decreases for higher values of viscous dissipation parameter $V d$. Also it is observed that at $\xi=0.50615$, the skin friction co-efficient $C_{f x}$ increases by $0.74 \%$ and the Nusselt number $N u_{x}$ decreases by $0.41 .75 \%$ as $V d$ increases from 0.0 to 4.0 .

Figs. 7(a) and 7(b) deal with the effect of Prandtl number on the skin friction and heat transfer co-efficient against $\xi$ with $V d=2.0, R d=1.0, Q=0.4$. Here, as the Prandtl number $\operatorname{Pr}$ increases, the skin friction coefficient $C_{f x}$ increases up to a certain level at $\xi=0.71588$ from the position of $\xi$ and then decreases gradually. It is seen that in Fig. 7(b) the rate of heat transfer $N u_{x}$ decreases with the increase of Prandtl number. This is expected because when Prandtl number $\operatorname{Pr}$ increases the skin friction coefficient and the rate of heat transfer both decrease due to viscosity.

The variation of the local skin friction co-efficient $C_{f x}$ and the local rate of heat transfer for different values of $R d$ with $V d=2.0, \operatorname{Pr}=0.72$ and $Q=0.4$ at different position of $\xi$ are illustrated in Figs. 8 (a) and 8(b), respectively. It is observed from Fig. 8 (a) that skin friction co-efficient $C_{f x}$ increases along the upward direction of $\xi$. This is to be expected because the higher value of the radiation parameter accelerates the fluid flow and increases the temperature as mentioned in Figs. 4 (a) and 4(b). With the increasing values of radiation parameter $R d$ the heat transfer co-efficient increases drastically to the surface of the sphere. It can also be seen that at $\xi$ $=0.50615$ both the skin friction co-efficient and the Nusselt number $N u_{x}$ increases by $18.15 \%$ and $412.74 \%$ as $R d$ increases from 0.0 to 1.0 .

From Figs. 9(a) and 9(b), we see that the effect of heat generation parameter $Q$ on the skin friction co-efficient $C_{f x}$ and rate of heat transfer against $\xi$ with $V d=2.0, \operatorname{Pr}=0.72$ and $R d=1.0$. The effect of $Q$ leads to a increase in the local skin friction co-efficient $C_{f x}$ but decreases monotonically in the local Nusselt number $N u_{x}$. These are expected, since the heat generation mechanism creates a layer of hot fluid flow near the surface of the sphere and finally the resultant temperature of the fluid exceeds the surface temperature. Owing to the enhanced temperature, the viscosity of the fluid increases and the corresponding skin friction co-efficient increases. When internal heat is generated the skin friction is increased. At $\xi=0.50615$ the skin friction co-efficient increases by $6.93 \%$ and the Nusselt number decreases by $78.36 \%$ as $Q$ increases from 0.2 to 0.4 .

\section{Comparison with previous work and program validation}

A comparison of the surface temperature and local skin friction coefficient obtained in the present work for viscous dissipation parameter $V d=0.0,1.0,2.0,3.0,4.0$ in presence of Prandtl number $\operatorname{Pr}=0.72$, radiation parameter $R d=1.0$ and heat generation parameter $Q=0.4$ has been shown in Table 1 . We also have compared our present numerical result for Nusselt number $N u_{x}$ in case of the Prandtl number $\operatorname{Pr}=0.7$ and 7 with Nazar et al. (2002) and Molla et al. (2005) respectively. It is clearly seen that there is an excellent agreement among the respective results.

Table 1: Comparison of the present numerical results of Skin friction coefficient and rate of heat transfer with $\mathrm{Pr}$ $=0.72, R d=1.0$ and $\mathrm{Q}=0.4$ against $\xi$.

\begin{tabular}{|l|l|l|l|l|l|l|l|l|l|l|}
\hline$\xi$ & \multicolumn{3}{l}{$V d=0.0$} & \multicolumn{2}{l|}{$V d=1.0$} & \multicolumn{2}{l|}{$V d=2.0$} & \multicolumn{2}{l|}{$V d=3.0$} & \multicolumn{2}{l|}{$V d=4.0$} \\
\hline & $C_{f x}$ & $N u_{x}$ & $C_{f x}$ & $N u_{x}$ & $C_{f x}$ & $N u_{x}$ & $C_{f x}$ & $N u_{x}$ & $C_{f x}$ & $N u_{x}$ \\
\hline 0 & 0.00 & 0.68 & 0.00 & 0.68 & 0.00 & 0.68751 & 0.00 & 0.68 & 0.00 & 0.68 \\
\hline$\pi / 6$ & 0.68 & 0.65 & 0.52 & 0.58 & 0.52 & 0.51 & 0.53 & 0.43 & 0.53 & 0.36 \\
\hline$\pi / 3$ & 1.07 & 0.55 & 0.96 & 0.28 & 0.97 & 0.02 & 0.98 & -0.25 & 0.98 & -0.54 \\
\hline$\pi / 2$ & 1.12 & 0.37 & 1.25 & -0.12 & 1.26 & -0.64 & 1.28 & -1.18 & 1.30 & -1.76 \\
\hline
\end{tabular}


Table 2: Comparison of the present numerical results of Nusselt number $N u_{x}$ with Prandtl number $\operatorname{Pr}=0.7$ and 7.0, $R d=1.0$ and $\mathrm{Q}=0.4$ against $\xi$.

\begin{tabular}{|c|c|c|c|c|c|c|}
\hline \multirow{2}{*}{$\begin{array}{c}\xi \\
\text { in degree }\end{array}$} & \multicolumn{3}{|c|}{$P r=0.7$} & \multicolumn{3}{|c|}{$\operatorname{Pr}=7.0$} \\
\hline & $\begin{array}{c}\text { Nazar } \\
\text { et al. }(2002)\end{array}$ & $\begin{array}{c}\text { Molla } \\
\text { et al. }(2005)\end{array}$ & $\begin{array}{c}\text { Present } \\
\text { work }\end{array}$ & $\begin{array}{c}\text { Nazar } \\
\text { et al. }(2002)\end{array}$ & $\begin{array}{c}\text { Molla } \\
\text { et al. }(2005)\end{array}$ & $\begin{array}{c}\text { Present } \\
\text { work }\end{array}$ \\
\hline 0 & 0.4576 & 0.4576 & 0.4575 & 0.9595 & 0.9582 & 0.9490 \\
\hline 10 & 0.4565 & 0.4564 & 0.4564 & 0.9572 & 0.9558 & 0.9560 \\
\hline 20 & 0.4533 & 0.4532 & 0.4533 & 0.9506 & 0.9492 & 0.9506 \\
\hline 30 & 0.4480 & 0.4479 & 0.4480 & 0.9397 & 0.9383 & 0.9397 \\
\hline 40 & 0.4405 & 0.4404 & 0.4406 & 0.9239 & 0.9231 & 0.9240 \\
\hline 50 & 0.4308 & 0.4307 & 0.4310 & 0.9045 & 0.9034 & 0.9047 \\
\hline 60 & 0.4308 & 0.4307 & 0.4310 & 0.8801 & 0.8791 & 0.8807 \\
\hline 70 & 0.4189 & 0.4188 & 0.4195 & 0.8510 & 0.8501 & 0.8513 \\
\hline 80 & 0.3879 & 0.3877 & 0.3882 & 0.8168 & 0.8161 & 0.8171 \\
\hline 90 & 0.3684 & 0.3683 & 0.3688 & 0.7774 & 0.7768 & 0.7778 \\
\hline
\end{tabular}

\section{Conclusion}

In this paper effects of viscous dissipation on natural convection flow over a sphere in the presence of radiation and heat generation have been studied numerically. Implicit finite difference method together with Keller box scheme is employed to integrate the equations governing the flow. Comparison with previously published work is performed and excellent argument has been observed. From the present numerical investigation, following conclusions may be drawn:

- The velocity within the boundary layer increases for the decreasing values of $\operatorname{Pr}$ and for the increasing values of $Q$ and $R d$.

- The temperature within the boundary layer increases for the increasing values of $Q$ and $R d$ and for the decreasing values of $\mathrm{Pr}$.

- The local skin friction coefficient decreases for the increasing values of $\operatorname{Pr}$ and increases for the increasing values of $Q$ and $R d$.

- An increase in the values of $\operatorname{Pr}$ and $R d$ leads to an increase in the surface temperature. On the other hand, this decreases for the increasing values of $Q$.

- For the increased value of viscous dissipation parameter $V d$, the velocity and the temperature profiles increase monotonically.

- Significant effects are found in skin friction and rate of heat transfer for the Prandtl number and heat generation parameters.

- The local skin friction coefficient and heat transfer distribution are clearer than the velocity and temperature profiles.

\section{Reference}

Gebhart, B. (1962): Effects of viscous dissipation in natural convection, Journal of Fluid Mechanics, Vol. 14, pp. 225-232. doi: $10.1017 / \mathrm{S} 0022112062001196$

Gebhart, B. and Mollendorf, J. (1969): Viscous dissipation in external natural convection flows, Journal of Fluid Mechanics, Vol.38, pp. 97-107. doi:10.1017/S0022112069000061

Keller, H.B.(1978): Numerical methods in boundary layer theory, Annual Rev. Fluid Mechanics, pp. 417-433. doi:10.1146/annurev.fl.10.010178.002221

Cebeci, T. and Bradshaw, P. (1984): Physical and Computational Aspects of Convective Heat Transfer, Springer, New York. doi.wiley.com/10.1002/zamm.19860661123

Siegel, R. and Howell, J.R. (1972): Thermal Radiation Heat Transfer, McGraw-Hill, New York. doi: $10.1002 /$ zamm.201290025 
Zakerullah, M., (1972): Viscous dissipation and pressure work affects in axisymmetric natural convection flows, Journal of Bangladesh Math. Soc. (Ganit) Vol. 2. No.1, pp.43- 48.

Huang, M. J. and Chen, C. K., (1987): Laminar free convection from a sphere with blowing and suction, Journal of Heat Transfer,Vol.109, pp.529-532. doi:10.1115/1.2910600

Sutton, G.P and T. and Biblarz,O.,(2001): Rocket Propulsion Elements, Seventh edition, John Wiley \& Sons.

Nazar, R., Amin, N., Grosan,T. and Pop , I. (2002): Free convection boundary layer on isothermal sphere in a micropolar fluid, Heat Transfer,29,Proceeding of the12th International conference .

Nazar, R., Amin, N., Grosan, T. and Pop, I. (2002): Free convection boundary layer on isothermal sphere in a micropolar fluid, Int.Comm. Heat Mass Transfer, Vol. 29 , No.3, pp.377-386. doi: 10.1007/s11012-011-9510-5

Molla, M. M., Hossain, M. A. and Yao, L .S. (2004): Natural convection flow along a vertical wavy surface with heat generation/absorption, International Journal of Thermal Science, Vol. 43, pp157-163. doi:10.1016/j.ijthermalsci.2003.04.001

Molla, M. M., Taher, M. A., Chowdhury, M. M. K. and Hossain, M. A. (2005): Magneto hydrodynamic natural convection flow on a sphere in presence of heat generation, Nonlinear Analysis: Modeling and Control, Vol. 10, No. 4, pp. 349-363. doi: 10.1007/s00707-006-0373-0

Alam, M. M., Alim, M. A., and Chowdhury, M. M. K. (2006): Effect of pressure stress work \& viscous dissipation in natural convection flow along a vertical flat plate with heat conduction, Journal of Naval Architecture and Marine Engineering,Vol. 3, No. 2, pp.69-76. doi: 10.3329/jname.v3i2.921

Molla, M. M., Hossain, M. A., Paul and Manosh. C. ((2006): Natural convection flow from an isothermal horizontal circular cylinder in presence of heat generation, International Journal of Engineering Science, 44(1314):949-958. doi:10.1016/j.jijengsci.2006.05.002

Alam, M. M., Alim, M. A., and Chowdhury, M. M. K. (2006): Viscous dissipation effects on MHD natural convection flow along a sphere, Journal of Mechanical Engineering, Vol.36, pp.44. doi: 10.3329/jme.v36i0.810

Alam, M. M., Alim, M .A., and Chowdhury, M. M. K. (2007): Viscous dissipation effects on MHD natural convection flow over a sphere in the presence of heat generation, Nonlinear Analysis: Modelling and Control, Vol. 12, No 4, pp. 447-459.

Akhter, Tahmina and Alim, M.A. (2008): Effects of radiation on natural convection flow around a sphere with uniform surface heat flux, Journal of Mechanical Engineering, vol.ME39, No.1.

Alim M. A., Rahman, M. M. and Aktar, S. (2008), Viscous dissipation effects on natural convection flow along a sphere with radiation heat loss, Proceeding of the the $4^{\text {th }}$ BSME-ASME International Conference on Thermal Engineering (27-29 December), Dhaka, Bangladesh.

Miraj, M. M., Alim, M. A. and Mamun, M. A. H. (2010): Effect of radiation on natural convection flow on a sphere in presence of heat generation, International Communications in Heat and Mass Transfer,Vol.37, pp.660665.

Tania, S. K. and Samad, M. A. (2010): Effects of radiation, heat generation and viscous dissipation on MHD free convection flow along a stretching sheet, Research Journal of Applied Science, Engineering and Technology 2(4),pp. 368-377.

Ali, M. M., Alim, M. A. and Andallah, L. S. (2011): Conjugate effects of Radiation and Joule Heating on Magnetohydrodynamic Free Convection Flow along a Sphere with Heat Generation, American Journal of Computational Mathematics,Vol.1,pp. 18-25. doi:10.4236/ajcm.2011.11003

Miraj, M. M., Alim, M. A. and Mamun, M. A. H. (2011): Effects of Pressure work on natural convection flow around a sphere with heat generation, International Communications in Heat and Mass Transfer, Vol. 15, No. 3, 287-298

Ibrahim, S. M. (2013): Chemical reaction and radiation effects on MHD free convection flow along a stretching surface with viscous dissipation and heat generation, Advances in Applied Science Research, Pelagia Research Library,Vol.4, No.1, pp.371-382. 